\title{
How to (Properly) Evaluate Cross-Lingual Word Embeddings: On Strong Baselines, Comparative Analyses, and Some Misconceptions
}

\author{
Goran Glavaš ${ }^{1}$, Robert Litschko ${ }^{1}$, Sebastian Ruder ${ }^{2,3}$, and Ivan Vulić ${ }^{4,5}$ \\ ${ }^{1}$ Data and Web Science Group, University of Mannheim, Germany \\ ${ }^{2}$ Insight Research Centre, National University of Ireland, Galway, Ireland \\ ${ }^{3}$ Aylien Ltd., Dublin, Ireland \\ ${ }^{4}$ Language Technology Lab, University of Cambridge, UK ${ }^{5}$ PolyAI Ltd., UK \\ \{goran, rlitschk\} @informatik.uni-mannheim.de, \\ sebastian@ruder.io, iv250@cam.ac.uk
}

\begin{abstract}
Cross-lingual word embeddings (CLEs) enable multilingual modeling of meaning and facilitate cross-lingual transfer of NLP models. Despite their ubiquitous usage in downstream tasks, recent increasingly popular projectionbased CLE models are almost exclusively evaluated on a single task only: bilingual lexicon induction (BLI). Even BLI evaluations vary greatly, hindering our ability to correctly interpret performance and properties of different CLE models. In this work, we make the first step towards a comprehensive evaluation of cross-lingual word embeddings. We thoroughly evaluate both supervised and unsupervised CLE models on a large number of language pairs in the BLI task and three downstream tasks, providing new insights concerning the ability of cutting-edge CLE models to support cross-lingual NLP. We empirically demonstrate that the performance of CLE models largely depends on the task at hand and that optimizing CLE models for BLI can result in deteriorated downstream performance. We indicate the most robust supervised and unsupervised CLE models and emphasize the need to reassess existing baselines, which still display competitive performance across the board. We hope that our work will catalyze further work on CLE evaluation and model analysis.
\end{abstract}

\section{Introduction and Motivation}

Following the ubiquitous use of word embeddings in monolingual NLP tasks, many researchers have broadened their interest towards cross-lingual word embeddings (CLEs). CLE models learn vectors of words in two or more languages and represent them in a shared cross-lingual word vector space, where words with similar meanings obtain similar vectors, irrespective of their language. Owing to this property, CLEs hold promise to support cross-lingual NLP by enabling multilingual model- ing of meaning and facilitating cross-lingual transfer for downstream NLP tasks and resource-poor languages. They serve as an invaluable source of (cross-lingual) knowledge in tasks such as bilingual lexicon induction (Mikolov et al., 2013; Heyman et al., 2017), document classification (Klementiev et al., 2012), information retrieval (Vulić and Moens, 2015), dependency parsing (Guo et al., 2015), sequence labeling (Zhang et al., 2016; Mayhew et al., 2017), and machine translation (Artetxe et al., 2018c; Lample et al., 2018), among others.

Earlier work typically induces CLEs by leveraging bilingual supervision from multilingual corpora aligned at the level of sentences (Klementiev et al., 2012; Zou et al., 2013; Hermann and Blunsom, 2014; Luong et al., 2015; Gouws et al., 2015, inter alia) and documents (Søgaard et al., 2015; Vulić and Moens, 2016; Levy et al., 2017, inter alia). A recent trend, however, are the so-called projectionbased approaches ${ }^{1}$ that post-hoc align pre-trained monolingual embeddings. Their popularity stems from their competitive performance coupled with a conceptually simple design, which requires only cheap bilingual supervision (Ruder et al., 2018b). In particular, they demand word-level supervision from seed translation dictionaries that span several thousand word pairs (Mikolov et al., 2013; Faruqui and Dyer, 2014; Huang et al., 2015), but it has also been shown that reliable projections can be bootstrapped from small dictionaries of 50-100 pairs (Vulić and Korhonen, 2016; Zhang et al., 2016), identical strings and cognates (Hauer et al., 2017; Smith et al., 2017; Søgaard et al., 2018), and shared numerals (Artetxe et al., 2017).

In addition, recent work has leveraged topological similarities between monolingual vector spaces to introduce fully unsupervised projectionbased CLE approaches that do not require any

\footnotetext{
${ }^{1}$ In the literature the methods are sometimes referred to as mapping-based CLE approaches or offline approaches.
} 
bilingual supervision (Zhang et al., 2017; Conneau et al., 2018a; Artetxe et al., 2018b; Alvarez-Melis and Jaakkola, 2018). Being conceptually attractive, such weakly supervised and unsupervised CLEs have taken the field by storm recently (Conneau et al., 2018a; Grave et al., 2018; Dou et al., 2018; Doval et al., 2018; Hoshen and Wolf, 2018; Joulin et al., 2018; Kim et al., 2018; Chen and Cardie, 2018; Mukherjee et al., 2018; Nakashole, 2018; Xu et al., 2018; Alaux et al., 2019).

Regardless of their underlying modeling assumptions as well as data and supervision requirements, all CLE models are directly comparable as they produce the same "end product": a shared cross-lingual word vector space. Yet, a proper and comprehensive evaluation of recent CLE models is missing. Limited CLE evaluations impede comparative analyses and even lead to inadequate conclusions, as the models are typically overfitted to a single task.

Whereas early CLE models (Klementiev et al., 2012; Hermann and Blunsom, 2014) were evaluated in downstream tasks (primarily cross-lingual text classification), a large body of recent work is judged exclusively on the task of bilingual lexicon induction (BLI). This limits our understanding of CLE methodology as: 1) BLI is an intrinsic task, and it has been shown that word translation performance and downstream performance on text classification and parsing tend to correlate poorly (Ammar et al., 2016); 2) We argue that BLI is not the main reason why we induce cross-lingual embedding spaces - rather, we seek to employ CLEs to tackle multilinguality and language transfer in downstream tasks. Therefore, it is not at all clear whether and to which extent BLI performance of (projection-based) CLE models correlates with various downstream tasks. In other words, previous research does not evaluate the true capacity of CLE models to support cross-lingual NLP.

Downstream evaluations aside, it is virtually impossible to directly compare all recently proposed mapping-based CLE models based on their published BLI results due to the lack of a common evaluation protocol: different papers consider different language pairs and employ different training and evaluation dictionaries. Furthermore, there is a surprising lack of testing BLI results for statistical significance. The mismatches in evaluation yield partial conclusions and inconsistencies: on the one hand, some unsupervised models (Artetxe et al., 2018b; Hoshen and Wolf, 2018; Alvarez-
Melis and Jaakkola, 2018) are reported to outperform (or at least perform on par with) previous best-performing supervised CLE models (Artetxe et al., 2017; Smith et al., 2017). On the other hand, the most recent supervised approaches (Doval et al., 2018; Joulin et al., 2018) report further performance gains, surpassing unsupervised models.

Supervised projection-based CLEs commonly require only small to moderately sized translation dictionaries (e.g. $1 \mathrm{~K}-5 \mathrm{~K}$ word translation pairs) and can also be bootstrapped from even smaller dictionaries. Such supervision data are easily obtainable for most language pairs. ${ }^{2}$ Therefore, despite their attractive zero-supervision setup, we perceive unsupervised CLE models practically justified only if such models can, contrary to the intuition, indeed outperform supervised projection-based CLEs.

Contributions. In this work, we provide a comprehensive comparative evaluation of a wide range of state-of-the-art-both supervised and unsupervised-projection-based CLE models. Our evaluation benchmark encompasses BLI and three cross-lingual downstream tasks of different nature: document classification (CLDC), information retrieval (CLIR), and natural language inference (XNLI). We unify evaluation protocols for all the representative models in our comparison, and conduct experiments on a large set of 28 language pairs that span diverse language types.

In addition to providing a unified testbed for guiding CLE research, we primarily aim at answering the following two research questions: 1) Is BLI performance a good predictor of downstream performance for projection-based CLE models? 2) Can unsupervised CLE models indeed outperform their supervised counterparts? In many of our experiments, the simplest among the evaluated CLE methods outperform their more intricate competitors. We find that overfitting to BLI may severely hurt downstream performance, indicating that BLI evaluation should always be coupled with downstream evaluations in order to paint a more informative picture of CLE models' properties.

\footnotetext{
${ }^{2}$ One could argue that if a small word translation dictionary cannot be obtained for a pair of languages, one is probably dealing with truly under-resourced languages for which it would be difficult to obtain a large corpus required to train reliable monolingual embeddings in the first place. Furthermore, there are initiatives in typological linguistics research such as the ASJP database (Wichmann et al., 2018), which offers 40-item word lists denoting the same set of concepts in all the world's languages: https://as jp.clld.org/. Indirectly, such lists can offer the initial seed supervision.
} 


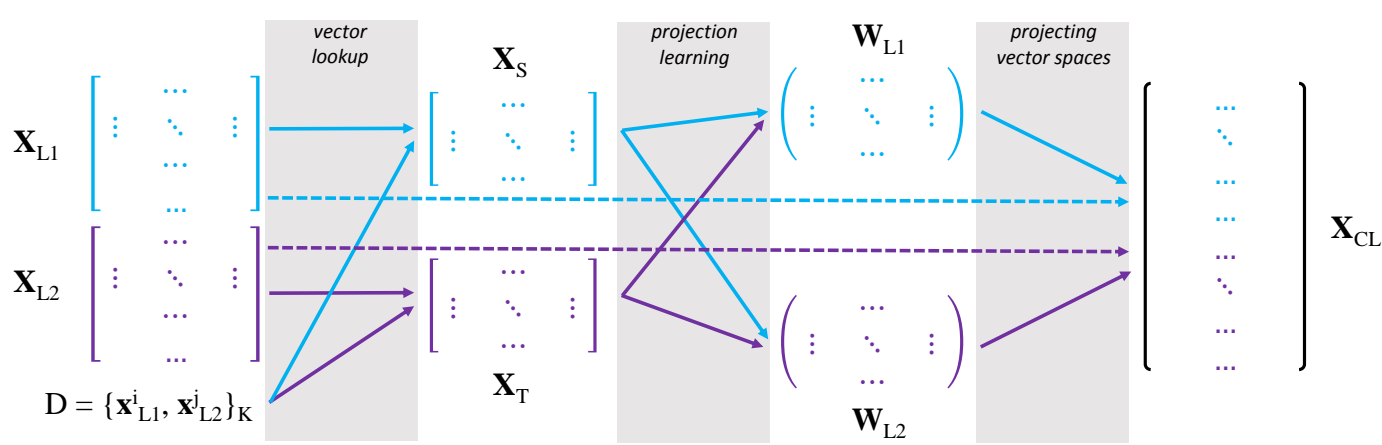

Figure 1: A general framework for post-hoc projection-based induction of cross-lingual word embeddings.

\section{Projection-Based Cross-Lingual Word Embeddings: Methodology}

In contrast to the more recent unsupervised models, CLE models typically require aligned words, sentences, or documents to learn a shared vector space. Supervised CLE models based on sentenceand document-aligned texts have been extensively studied and categorized according to the nature of required bilingual supervision in previous work (Vulić and Korhonen, 2016; Upadhyay et al., 2016; Ruder et al., 2018b). For a systematic overview of these earlier (resource-demanding) CLE models, we refer the reader to the survey papers. Current CLE research is almost exclusively focused on projection-based CLE models; they are therefore also the focal point of our comparative study. ${ }^{3}$

\subsection{Projection-Based Framework}

The goal is to learn a projection between independently trained monolingual word vector spaces. The mapping is sought using a seed bilingual lexicon, provided beforehand or extracted without supervision. A general post-hoc projection-based CLE induction framework is depicted in Figure 1. Let $\mathbf{X}_{L 1}$ and $\mathbf{X}_{L 2}$ be the monolingual embedding spaces of the two languages with respective vocabularies $V_{L 1}$ and $V_{L 2}$. All projection-based CLE models encompass the following steps:

Step 1: Construct the seed translation dictionary $D=\left\{\left(w_{L 1}^{i}, w_{L 2)}^{j}\right\}_{k=1}^{K}\right.$ containing $K$ word pairs. Supervised models simply use an external dictionary; unsupervised models induce $D$ automatically, usually assuming (approximate) isomorphism between monolingual spaces.

Step 2: Create aligned monolingual subspaces (i.e.,

\footnotetext{
${ }^{3}$ Since these methods a) are not bound to any particular word embedding model (i.e., they are fully agnostic to how we obtain monolingual vectors) and b) they do not require any multilingual corpora, they lend themselves to a wider spectrum of languages than the alternatives (Ruder et al., 2018b).
}

matrices) $\mathbf{X}_{S}=\left\{\mathbf{x}_{L 1}^{i}\right\}_{k=1}^{K}$ and $\mathbf{X}_{T}=\left\{\mathbf{x}_{L 2}^{j}\right\}_{k=1}^{K}$ by retrieving monolingual vectors of words from the translation dictionary: vectors of $\left\{w_{L 1}^{i}\right\}_{k=1}^{K}$ from $\mathbf{X}_{L 1}$ and vectors of $\left\{w_{L 2}^{j}\right\}_{k=1}^{K}$ from $\mathbf{X}_{L 2}$.

Step 3: Learn to project to the joint cross-lingual space using the word-aligned matrices $\mathbf{X}_{S}$ and $\mathbf{X}_{T}$. In the general case, we learn two projection matrices, $\mathbf{W}_{L 1}$ and $\mathbf{W}_{L 2}$, projecting respective vectors from $\mathbf{X}_{L 1}$ and $\mathbf{X}_{L 2}$ to the shared space $\mathbf{X}_{C L}$. Most models, however, directly project source language vectors from $\mathbf{X}_{L 1}$ to the target language space $\mathbf{X}_{L 2}$ (i.e., $\mathbf{W}_{L 2}=I$ and $\mathbf{X}_{C L}=\mathbf{X}_{L 1} \mathbf{W}_{L 1} \cup \mathbf{X}_{L 2}$ ).

\subsection{Projection-Based CLE Models}

We evaluate a range of both supervised and unsupervised projection-based CLE models. While supervised models (Mikolov et al., 2013; Smith et al., 2017; Ruder et al., 2018a; Joulin et al., 2018) learn the projections using existing dictionaries, unsupervised models first induce seed dictionaries without bilingual data. We include unsupervised models with diverse dictionary induction strategies: adversarial learning (Conneau et al., 2018a), similarity-based heuristics (Artetxe et al., 2018b), PCA (Hoshen and Wolf, 2018), and optimal transport (Alvarez-Melis and Jaakkola, 2018). We next briefly describe the CLE models in our evaluation.

Canonical Correlation Analysis (CCA). Faruqui and Dyer (2014) use CCA to project $\mathbf{X}_{L 1}$ and $\mathbf{X}_{L 2}$ into a shared space $\mathbf{X}_{C L}$. CCA learns both $W_{L 1}$ and $W_{L 1}$, one for mapping each of the input spaces to the shared space. We evaluate CCA as a simple supervised baseline model that has mostly been neglected in recent BLI evaluations.

Solving the Procrustes Problem. In their seminal projection-based CLE work, Mikolov et al. (2013) cast the problem as learning the projection $\mathbf{W}_{L 1}$ that minimizes the Euclidean distance between the projection of $\mathbf{X}_{S}$ and $\mathbf{X}_{T}$ : 


$$
\mathbf{W}_{L 1}=\underset{\mathbf{W}}{\arg \min }\left\|\mathbf{X}_{L 1} \mathbf{W}-\mathbf{X}_{L 2}\right\|_{2}
$$

They also report non-linear neural projections yielding worse BLI performance than a linear map $\mathbf{W}_{L 1}$. Xing et al. (2015) later achieves further BLI gains by constraining $\mathbf{W}_{L 1}$ to an orthogonal matrix, ensuring that the topology of the original monolingual space is preserved. By imposing the orthogonality constraint, the optimization problem from Eq. (1) becomes the Procrustes problem, with the following closed-form solution (Schönemann, 1966):

$$
\begin{aligned}
\mathbf{W}_{L 1} & =\mathbf{U V}^{\top}, \text { with } \\
\mathbf{U} \boldsymbol{\Sigma} \mathbf{V}^{\top} & =S V D\left(\mathbf{X}_{T} \mathbf{X}_{S}^{\top}\right) .
\end{aligned}
$$

We use the linear map obtained as the solution to the Procrustes problem as the primary baseline in our evaluation (PROC). Furthermore, inspired by the bootstrapping self-learning procedures that some unsupervised models employ to (iteratively) augment the induced lexicon $D$ (Artetxe et al., 2018b; Conneau et al., 2018a), we propose a simple bootstrapping-based extension of the supervised PROC model (dubbed PROC-B). The intuition is that the bootstrapping approach can boost performance when smaller supervised lexicons are used. The procedure is summarized in Algorithm 1.

In each iteration, we use the translation lexicon $D$ to learn two unidirectional projections $-\mathbf{W}_{L 1}$ that projects the embedding space $\mathbf{X}_{L 1}$ of L1 to the embedding space $\mathbf{X}_{L 2}$ of L2 and $\mathbf{W}_{L 2}$ that, inversely, projects vectors from $\mathbf{X}_{L 2}$ to $\mathbf{X}_{L 1}$. We next create two sets of word translation pairs by comparing (1) $\mathbf{X}_{L 1} \mathbf{W}_{L 1}$ with $\mathbf{X}_{L 2}$ (denoted as $D_{12}$ ) and (2) $\mathbf{X}_{L 2} \mathbf{W}_{L 2}$ with $\mathbf{X}_{L 1}$ (denoted as $D_{21}$ ). We

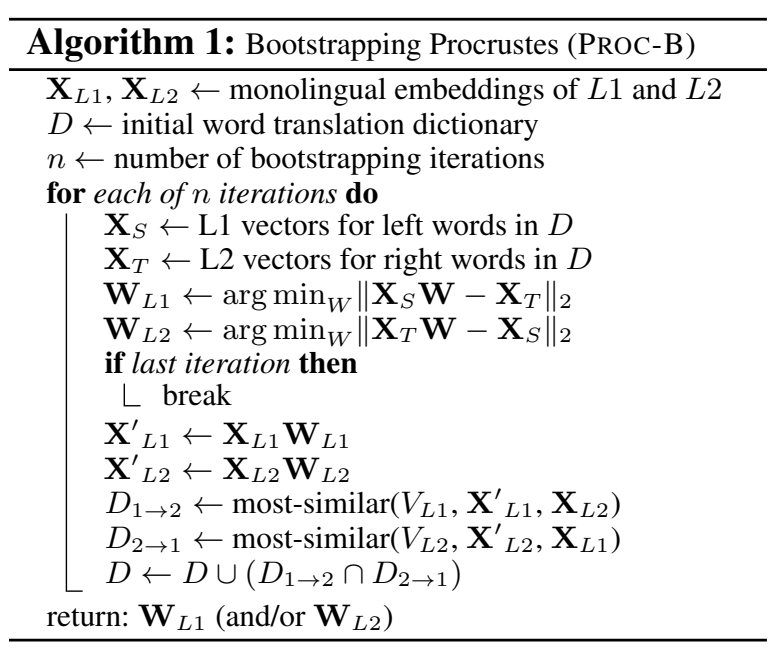

then augment the dictionary $D$ with mutual nearest neighbours we find: $D_{12} \cap D_{21}$.

Discriminative Latent-Variable (DLV). Ruder et al. (2018a) augment the seed supervised lexicon through Expectation-Maximization in a latentvariable model. The source words $\left\{w_{L 1}^{i}\right\}_{k=1}^{K}$ and target words $\left\{w_{L 2}^{j}\right\}_{k=1}^{K}$ are seen as a fully connected bipartite weighted graph $G=\left(E, V_{L 1} \cup\right.$ $\left.V_{L 2}\right)$ with edges $E=V_{L 1} \times V_{L 2}$. By drawing embeddings from a Gaussian distribution and normalizing them, the weight of each edge $(i, j) \in E$ is shown to correspond to the cosine similarity between vectors. In the E-step, a maximal bipartite matching is found on the sparsified graph using the Jonker-Volgenant algorithm (Jonker and Volgenant, 1987). In the M-step, a better projection $W_{L 1}$ is learned solving the Procrustes problem.

Ranking-Based Optimization (RCSLS). Joulin et al. (2018) optimize the projection matrix $\mathbf{W}_{L 1}$ by maximizing the cross-domain similarity local scaling (CSLS; Conneau et al., 2018a) score, instead of minimizing the Euclidean distances between projection of $\mathbf{X}_{S}$ and $\mathbf{X}_{T}$. CSLS is a modification of cosine similarity commonly used for BLI inference. Let $r\left(\mathbf{x}_{L 1}^{k} \mathbf{W}, \mathbf{X}_{L 2}\right)$ be the average cosine similarity of the projected vector $\mathbf{x}_{L 1}^{k} \mathbf{W}$ with its $N$ nearest neighbors from $\mathbf{X}_{L 2}$. Similarly, let $r\left(\mathbf{x}_{L 2}^{k}, \mathbf{X}_{L 1} \mathbf{W}\right)$ be the average cosine similarity of the target space vector $\mathrm{x}_{L 2}^{k}$ with its $N$ nearest neighbors from the projected source space $\mathbf{X}_{L 1} \mathbf{W}$. By relaxing the constraint that $\mathbf{W}_{L 1}$ is orthogonal, maximization of relaxed CSLS (dubbed RCSLS) becomes a convex optimization problem:

$$
\begin{aligned}
\mathbf{W}_{L 1} & =\underset{\mathbf{W}}{\arg \min } \frac{1}{K} \sum_{\substack{\mathbf{x}_{L 1}^{k} \in X_{S} \\
\mathbf{x}_{L 2}^{k} \in X_{T}}}-2 \cos \left(\mathbf{x}_{L 1}^{k} \mathbf{W}, \mathbf{x}_{L 2}^{k}\right) \\
& +r\left(\mathbf{x}_{L 1}^{k} \mathbf{W}, \mathbf{X}_{L 2}\right)+r\left(\mathbf{x}_{L 2}^{k}, \mathbf{X}_{L 1} \mathbf{W}\right)
\end{aligned}
$$

By maximizing (R)CSLS, this model is explicitly designed to induce a cross-lingual embedding space that performs well in the BLI task.

Adversarial Alignment (MUSE). Several CLE models initialize a seed bilingual lexicon solely from monolingal data using Generative Adversarial

\footnotetext{
${ }^{4}$ In preliminary experiments, we achieve best performance using only a single bootstrapping iteration. Even when starting with $D$ of $1 \mathrm{~K}$ entries, after the first iteration we find between $5 \mathrm{~K}$ and $10 \mathrm{~K}$ mutual nearest neighbours that yield a better mapping. In the second iteration already we obtain a much larger number of noisier (automatically generated) word translations, which do not lead to further performance gains.
} 
Networks (GANs; Goodfellow et al., 2014), with MUSE (Conneau et al., 2018a) being the most effective example. The generator component in MUSE is again the linear map $\mathbf{W}_{L 1}$. MUSE aims to improve the generator mapping $\mathbf{W}_{L 1}$ such that the discriminator (a binary classifier implemented as multi-layer perceptron) fails to distinguish between the vector sampled from the target language distribution $\mathbf{X}_{L 2}$ and the projected vector $\mathbf{X}_{L 1} \mathbf{W}_{L 1}$. MUSE then improves the GAN-induced mapping, through a refinement step very similar to the PROCB procedure. MUSE strongly assumes approximate isomorphism of monolingual spaces (Søgaard et al., 2018), often leading to poor GAN-based initialization, especially for pairs of distant languages.

Heuristic Alignment (VecMAP). Artetxe et al. (2018b) induce the initial seed lexicon $D$ using a heuristic that assumes that for a pair of translations, their monolingual similarity vectors (i.e, vectors of, e.g., cosine or CSLS, similarities with all words in L1/L2) are (approximately) equal. Seed translations are generated by finding nearest neighbors based on the similarity between monolingual similarity distributions of words. Next, they employ a self-learning bootstrapping procedure similar to MUSE. In addition to the different initialization, VECMAP critically relies on a number of empirically motivated enhancements that ensure its robustness. It adopts both multi-step pre-processing consisting of unit length normalization, mean centering, and ZCA whitening (Bell and Sejnowski, 1997) as well several post-processing steps: crosscorrelational re-weighting, de-whitening, and dimensionality reduction (Artetxe et al., 2018a). What is more, VECMAP critically relies on stochastic dictionary induction: at each iteration, some elements in the similarity matrix are set to 0 based on the probability that varies across iterations. This enables the model to escape poor local optima.

Iterative Closest Point Model (ICP). The unsupervised model of Hoshen and Wolf (2018) induces initial seed dictionary $D$ by projecting vectors of the $N$ most frequent words from both monolingual spaces to a lower-dimensional space with PCA. They then search for an optimal alignment between L1 words (vectors $\mathbf{x}_{1}^{i}$ ) and L2 words (vectors $\mathbf{x}_{2}^{j}$ ), assuming linear projections $\mathbf{W}_{1}$ and $\mathbf{W}_{2}$. Let $f_{1}(i)$ (vice versa $f_{2}(j)$ ) denote the index of the L2 word (vice versa L1 word) to which $\mathrm{x}_{1}^{i}$ (vice versa $\mathrm{x}_{2}^{j}$ ) is aligned. The optimization task is to find such translation matrices $\mathbf{W}_{1}$ and $\mathbf{W}_{2}$ that minimize the sum of Euclidean distances between optimally aligned vectors. They use Iterative Closest Point, a two-step iterative optimization algorithm that first fixes translation matrices $\mathbf{W}_{1}$ and $\mathbf{W}_{2}$ to find the optimal alignments and then use those alignments to update the translation matrices by minimizing:

$$
\begin{gathered}
\sum_{i}\left\|\mathbf{x}_{1}^{i} \mathbf{W}_{1}-\mathbf{x}_{2}^{f_{1}(i)}\right\|+\sum_{j}\left\|\mathbf{x}_{2}^{j} \mathbf{W}_{2}-\mathbf{x}_{1}^{f_{2}(j)}\right\|+ \\
\lambda \sum_{i}\left\|\mathbf{x}_{1}^{i}-\mathbf{x}_{1}^{i} \mathbf{W}_{1} \mathbf{W}_{2}\right\|+\lambda \sum_{j}\left\|\mathbf{x}_{2}^{j}-\mathbf{x}_{2}^{j} \mathbf{W}_{2} \mathbf{W}_{1}\right\|
\end{gathered}
$$

The second-line terms are cyclical consistency constraints forcing vectors round-projected to the other language space and back to remain unchanged. Next, they employ a dictionary bootstraping procedure based on mutual nearest neighbours (similar to PROC-B and MUSE) and produce the final projections by solving the Procrustes problem.

Gromov-Wasserstein Alignment Model (GWA). Observing that word embedding models employ metric recovery algorithms, Alvarez-Melis and Jaakkola (2018) cast CLE induction as an optimal transport problem based on the GromovWasserstein distance (with cosine distance as a cost measure). They first compute intra-language costs $\mathbf{C}_{L 1}=\cos \left(\mathbf{X}_{L 1}, \mathbf{X}_{L 1}\right)$ and $\mathbf{C}_{L 2}=$ $\cos \left(\mathbf{X}_{L 2}, \mathbf{X}_{L 2}\right)$ as well as inter-language similarities $\mathbf{C}_{12}=\mathbf{C}_{L 1}^{2} \mathbf{p} \mathbb{1}_{m}^{\top}+\mathbb{1}_{n} \mathbf{q}\left(\mathbf{C}_{L 2}^{2}\right)^{\top}$, with $\mathbf{p}$ and $\mathbf{q}$ as uniform probability distributions over $V_{L 1}$ and $V_{L 2}$, respectively. They then induce the initial projections by solving the Gromov-Wasserstein optimal transport problem with a fast iterative algorithm (Peyré et al., 2016), iteratively updating parameter vectors $\mathbf{a}$ and $\mathbf{b}$ :

$$
\mathbf{a}=\mathbf{p} \oslash \mathbf{K b}, \quad \mathbf{b}=\mathbf{q} \oslash \mathbf{K}^{\top} \mathbf{a},
$$

where $\oslash$ is element-wise division and $\mathbf{K}=$ $\exp \left(-\hat{\mathbf{C}}_{\Gamma} / \lambda\right)$ (with $\hat{\mathbf{C}}_{\Gamma}=\mathbf{C}_{12}-2 \mathbf{C}_{L 1} \Gamma \mathbf{C}_{L 2}^{\top}$ ). The alignment matrix $\Gamma=\operatorname{diag}(\mathbf{a}) \mathbf{K} \operatorname{diag}(\mathbf{b})$ is recomputed in each iteration. The final projection $\mathbf{W}_{L 1}$ is (again!) obtained by solving Procrustes using the final alignments in $\Gamma$ as supervision.

In sum, our brief overview points to the main high-level (dis)similarities of all projection-based CLE models: while they differ in the way the initial seed lexicon is extracted, most models (with CCA, PROC, and RCSLS as exceptions) are based on self-learning procedures that repeatedly solve the Procrustes problem from Eq. (2), typically on the trimmed vocabulary. In the final step, the fine-tuned linear map is applied on the full vocabulary. 


\section{Bilingual Lexicon Induction}

Bilingual lexicon induction has become the de facto standard evaluation task for projection-based CLE models. Given a shared CLE space, the task is to retrieve target language translations for a (test) set of source language words. A typical BLI evaluation in the recent literature reports comparisons with the well-known MUSE model on a few language pairs, all of which involve English as one of the languages. A comprehensive comparative BLI evaluation of a wider set of models conducted on a larger set of language pairs is still missing. In our evaluation, we include a much larger set of language pairs, including pairs in which neither of the languages is English. ${ }^{5}$ Furthermore, to allow both for a fair comparison between supervised models and for performance comparisons between different language pairs, we create training and evaluation dictionaries that are fully aligned across all evaluated language pairs. Finally, we also discuss other choices in existing BLI evaluations which are currently taken for granted: e.g., (in)appropriate evaluation metrics and lack of significance testing.

Language Pairs. Our evaluation comprises eight languages: Croatian (HR), English (EN), Finnish (FI), French (FR), German (DE), Italian (IT), Russian (RU), and Turkish (TR). For diversity, we selected two languages from three different IndoEuropean branches-Germanic (EN, DE), Romance (FR, IT), and Slavic (HR, RU) - as well as two nonIndo-European languages (FI from the Uralic family, and TR from the Turkic family). Creating all possible pairs between these eight languages results in a total of 28 language pairs under evaluation.

Monolingual Embeddings. Following prior work, we use 300-dimensional fastText embeddings (Bojanowski et al., 2017), ${ }^{6}$ pretrained on full Wikipedias of each language. We trim all vocabularies to the $200 \mathrm{~K}$ most frequent words.

Translation Dictionaries. We automatically created translation dictionaries using Google Translate similar to prior work (Conneau et al., 2018a). We selected the $20 \mathrm{~K}$ most frequent English words and automatically translated them to the other seven languages. We retained only tuples for which all

\footnotetext{
${ }^{5}$ To the best of our knowledge, all language pairs in existing BLI evaluations involved English as one of the languages (either source or target language)—with the exception of Estonian-Finnish used by Søgaard et al. (2018).

${ }^{6}$ https: / / fasttext.cc/docs/en/ crawl-vectors.html
}

translations were unigrams found in vocabularies of their respective monolingual embedding spaces, leaving us with $\approx 7 \mathrm{~K}$ tuples. We reserved $5 \mathrm{~K}$ tuples created from the more frequent English words for training, and the remaining $2 \mathrm{~K}$ tuples as test dictionaries. We also created two smaller training dictionaries, by selecting tuples corresponding to the $1 \mathrm{~K}$ and $3 \mathrm{~K}$ most frequent English words.

Evaluation Measures and Significance. BLI is cast as a ranking (i.e., retrieval) task; existing BLI evaluations employ $P @ k$ (precision at rank $k$, commonly with $k \in\{1,5,10\}$ ) for evaluation. We advocate the use of mean average precision (MAP) instead. ${ }^{7}$ While correlated with $P @ k$, MAP is more informative: unlike MAP, $P @ k$ treats all models that rank the correct translation below $k$ equally. ${ }^{8}$

The limited size of BLI test sets warrants statistical significance testing. Yet, a common case in BLI evaluation is to declare performance gains based purely on (rather limited) numeric performance increases (e.g., below 1-point $P @ 1$ ), without any significance analysis. We test the significance of BLI results by applying the two-tailed t-test with the Bonferroni correction (Dror et al., 2018).

\subsection{Results and Discussion}

Table 1 summarizes BLI performance over multiple language pairs. RCSLS (Joulin et al., 2018) displays the strongest BLI performance. This is not a surprising finding given that its learning objective is tailored for BLI in particular. RCSLS outperforms other supervised models (CCA, PROC, and DLV) trained with exactly the same dictionaries. Smith et al. (2017) already suggested that CCA and PROC display similar performance. We confirm that their performance, as well as the performance of DLV, are statistically indistinguishable, even at $\alpha=0.1$. Our bootstrapping PROC-B approach, significantly boosts the performance of PROC when using a small translation dictionary of $1 \mathrm{~K}$ pairs $(p<0.01)$. For the same $1 \mathrm{~K}$ training dictionary, PROC-B significantly outperforms RCSLS as well. Interestingly, for each supervised model, training on $5 \mathrm{~K}$ pairs does not significantly outscore the variant trained on $3 \mathrm{~K}$ pairs, whereas training on $3 \mathrm{~K}$ or $5 \mathrm{~K}$ pairs does significantly outperform the $1 \mathrm{~K}$ variants. This confirms a finding from prior work (Vulić

\footnotetext{
${ }^{7}$ In this setup with only one correct translation for each query, MAP is equivalent to mean reciprocal rank (MRR).

${ }^{8}$ For instance, when relying on $P @ 5$, a model that ranks the correct translation at rank 6 is equally penalized as the model that ranks it at rank $200 \mathrm{~K}$.
} 


\begin{tabular}{|c|c|c|c|c|}
\hline Model & Dict & All LPs & Filt. LPs & Succ. LPs \\
\hline \multicolumn{5}{|c|}{ Supervised } \\
\hline CCA & $1 \mathrm{~K}$ & .289 & .404 & $28 / 28$ \\
\hline $\mathrm{CCA}$ & $3 \mathrm{~K}$ & .378 & .482 & $28 / 28$ \\
\hline $\mathrm{CCA}$ & $5 \mathrm{~K}$ & .400 & .498 & $28 / 28$ \\
\hline$\overline{\mathrm{P}} \overline{\mathrm{RO}} \overline{\bar{C}}$ & $\overline{1} \bar{K}$ & $.2 \overline{9} \overline{9}$ &.$\overline{4} 1 \overline{1}$ & $\overline{2} 8 \overline{2} \overline{8}$ \\
\hline PROC & $3 \mathrm{~K}$ & .384 & .487 & $28 / 28$ \\
\hline PROC & $5 \mathrm{~K}$ & .405 & .503 & $28 / 28$ \\
\hline$\overline{\mathrm{P}} \overline{\mathrm{ROC}}-\overline{\mathrm{B}} \overline{\mathrm{B}}$ & $\overline{1} \bar{K}$ & $.3 \overline{7} 9^{-}$ &.$\overline{4} 8 \overline{5}$ & $\overline{2} 8 \overline{2} / \overline{8}$ \\
\hline PROC-B & $3 \mathrm{~K}$ & .398 & .497 & $28 / 28$ \\
\hline$\overline{\mathrm{D}} \overline{\mathrm{L}} \overline{\mathrm{V}}-$ & $\overline{1} \bar{K}$ & $.2 \overline{8} 9^{-}$ &.$\overline{4} 0 \overline{0}$ & $\overline{2} 8 \overline{2} 2 \overline{8}$ \\
\hline DLV & $3 \mathrm{~K}$ & .381 & .484 & $28 / 28$ \\
\hline DLV & $5 \mathrm{~K}$ & .403 & .501 & $28 / 28$ \\
\hline$\overline{\mathrm{R}} \overline{\mathrm{C}} \overline{\mathrm{SL}} \overline{\mathrm{S}}$ & $\overline{1} \bar{K}$ & $.3 \overline{3} \overline{1}$ &.$\overline{4} 4 \overline{1}$ & $\overline{2} 8 \overline{2} 2 \overline{8}$ \\
\hline RCSLS & $3 \mathrm{~K}$ & .415 & .511 & $28 / 28$ \\
\hline RCSLS & $5 \mathrm{~K}$ & .437 & .527 & $28 / 28$ \\
\hline \multicolumn{5}{|c|}{ Unupervised } \\
\hline VECMAP & - & .375 & .471 & $28 / 28$ \\
\hline MUSE & - & .183 & .458 & $13 / 28$ \\
\hline ICP & - & .253 & .424 & $22 / 28$ \\
\hline GWA & - & .137 & .345 & $15 / 28$ \\
\hline
\end{tabular}

Table 1: Summary of BLI performance (MAP). All LPs: average scores over all 28 language pairs; Filt. LP: average scores only over language pairs for which all models in evaluation yield at least one successful run; Succ. LPs: the number of language pairs for which we obtained at least one successful run. A run is considered successful if MAP $\geq 0.05$.

and Korhonen, 2016) suggesting that no significant improvements are to be expected from training linear maps on dictionaries larger than $5 \mathrm{~K}$ entries.

The results highlight VECMAP (Artetxe et al., 2018 b) as the most robust choice among all unsupervised models: besides being the only model to produce successful runs consistently for all language pairs, it also substantially outperforms all other unsupervised models. It is also the most effective unsupervised model on the subset of language pairs for which the other models produce successful runs. However, VECMAP is still significantly outperformed ( $p \leq 0.0002)$ by PROC-B trained on $1 \mathrm{~K}$ translation pairs and by all supervised models trained on $3 \mathrm{~K}$ and $5 \mathrm{~K}$ word pairs. These findings challenge unintuitive claims from recent work (Artetxe et al., 2018b; Hoshen and Wolf, 2018; Alvarez-Melis and Jaakkola, 2018) that the unsupervised CLE models are competitive to or even surpass supervised CLE models in the BLI task.

Table 2 shows the scores for a subset of 10 language pairs using a subset of models from Table $1 .^{9}$ As expected, all models work reasonably well for major languages - this is most likely due to a higher

\footnotetext{
${ }^{9}$ We provide full BLI results for all 28 language pairs and all models in the supplemental material (see appendix).
}

quality of the respective monolingual embeddings, which are pre-trained on much larger corpora. ${ }^{10}$ Language proximity also plays a critical role: on average, models achieve better BLI performance for languages from the same family (e.g., compare the results of HR-RU vs. HR-EN).

The gap between the best-performing supervised model (RCSLS) and the best-performing unsupervised model (VECMAP) is more pronounced for cross-family language pairs, especially those consisting of one Germanic and one Slavic or non-IndoEuropean language (e.g., 19 points MAP difference for EN-RU, 14 for DE-RU and EN-FI, 10 points for EN-TR and EN-HR, 9 points for DE-FI and EN-HR). We suspect that this is due to heuristics based on intra-language similarity distributions, employed by Artetxe et al. (2018b) to induce an initial translation dictionary, being less effective the more distant the languages in the pair are.

\section{Downstream Evaluation}

We now test projection-based CLE models in extrinsic cross-lingual applications, moving beyond the limiting BLI evaluation. We select three diverse cross-lingual downstream tasks: 1) crosslingual transfer for natural language inference (XNLI), a sentence-level language understanding task; 2) cross-lingual document classification (CLDC); which requires only shallow (i.e., lexicallevel or topical) meaning modeling, and 3) crosslingual information retrieval (CLIR), an unsupervised ranking task that relies on detecting coarser semantic relatedness.

\subsection{Natural Language Inference}

Large training corpora for NLI exist only in English (Bowman et al., 2015; Williams et al., 2018). Recently, Conneau et al. (2018b) released a multilingual XNLI corpus created by translating dev and test sets of the MultiNLI corpus (Williams et al., 2018) to 15 languages, hoping to foster research on cross-lingual sentence understanding.

Evaluation Setup. The multilingual XNLI corpus covers 5 out of our 8 languages used previously for BLI: EN, DE, FR, RU, and TR. Our XNLI evaluation setup is straightforward: we train a well-known and robust neural NLI model, Enhanced Sequential Inference Model (ESIM; Chen et al., 2017) as im-

\footnotetext{
${ }^{10}$ For instance, EN Wikipedia is approximately 3 times larger than DE and RU Wikipedias, 19 times larger than FI Wikipedia and 46 times larger than HR Wikipedia.
} 


\begin{tabular}{llcccccccccc}
\hline Model & Dict & EN-DE & IT-FR & HR-RU & EN-HR & DE-FI & TR-FR & RU-IT & FI-HR & TR-HR & TR-RU \\
\hline PROC & $1 \mathrm{~K}$ & 0.458 & 0.615 & 0.269 & 0.225 & 0.264 & 0.215 & 0.360 & 0.187 & 0.148 & 0.168 \\
PROC & $5 \mathrm{~K}$ & 0.544 & 0.669 & 0.372 & 0.336 & 0.359 & 0.338 & 0.474 & 0.294 & 0.259 & 0.290 \\
PROC-B & $1 \mathrm{~K}$ & 0.521 & 0.665 & 0.348 & 0.296 & 0.354 & 0.305 & 0.466 & 0.263 & 0.210 & 0.230 \\
RCSLS & $1 \mathrm{~K}$ & 0.501 & 0.637 & 0.291 & 0.267 & 0.288 & 0.247 & 0.383 & 0.214 & 0.170 & 0.191 \\
RCSLS & $5 \mathrm{~K}$ & 0.580 & 0.682 & 0.404 & 0.375 & 0.395 & 0.375 & 0.491 & 0.321 & 0.285 & 0.324 \\
\hline VECMAP & - & 0.521 & 0.667 & 0.376 & 0.268 & 0.302 & 0.341 & 0.463 & 0.280 & 0.223 & 0.200 \\
\hline Average & - & 0.520 & 0.656 & 0.343 & 0.294 & 0.327 & 0.304 & 0.440 & 0.260 & 0.216 & 0.234 \\
\hline
\end{tabular}

Table 2: BLI performance (MAP) with a selection of models on a subset of evaluated language pairs.

\begin{tabular}{lcccccc}
\hline Model & Dict & EN-DE & EN-FR & EN-TR & EN-RU & Avg \\
\hline \multicolumn{2}{l}{ Supervised } \\
\hline PROC & $1 \mathrm{~K}$ & 0.561 & 0.504 & 0.534 & 0.544 & 0.536 \\
PROC & $5 \mathrm{~K}$ & 0.607 & 0.534 & 0.568 & 0.585 & $\mathbf{0 . 5 7 4}$ \\
PROC-B & $1 \mathrm{~K}$ & 0.613 & 0.543 & 0.568 & 0.593 & $\mathbf{0 . 5 7 9}$ \\
PROC-B & $3 \mathrm{~K}$ & 0.615 & 0.532 & 0.573 & 0.599 & $\mathbf{0 . 5 8 0}$ \\
DLV & $5 \mathrm{~K}$ & 0.614 & 0.556 & 0.536 & 0.579 & $\mathbf{0 . 5 7 1}$ \\
RCSLS & $1 \mathrm{~K}$ & 0.376 & 0.357 & 0.387 & 0.378 & 0.374 \\
RCSLS & $5 \mathrm{~K}$ & 0.390 & 0.363 & 0.387 & 0.399 & 0.385 \\
\hline Unupervised & & & & & \\
\hline VECMAP & - & 0.604 & 0.613 & 0.534 & 0.574 & $\mathbf{0 . 5 8 1}$ \\
MUSE & - & 0.611 & 0.536 & $0.359^{*}$ & $0.363 *$ & 0.467 \\
ICP & - & 0.580 & 0.510 & $0.400^{*}$ & 0.572 & 0.516 \\
GWA & - & $0.427 *$ & $0.383 *$ & $0.359 *$ & $0.376^{*}$ & 0.386 \\
\hline
\end{tabular}

Table 3: XNLI performance (test set accuracy). Bold: highest scores, with mutually insignificant differences according to the non-parametric shuffling test (Yeh, 2000). Asterisks denote language pairs for which CLE models could not yield successful runs in the BLI task.

plemented by Williams et al. (2018), ${ }^{11}$ on the large English MultiNLI corpus, using EN word embeddings from a shared EN-L2 $(\mathrm{L} 2 \in\{\mathrm{DE}, \mathrm{FR}, \mathrm{RU}, \mathrm{TR}$ \}) embedding space. We then evaluate the model on the L2 XNLI test set by feeding L2 embeddings from the shared space. ${ }^{12,13}$

Results and Discussion. XNLI accuracy scores are summarized in Table 3. The mismatch between BLI and XNLI performance is most obvious for RCSLS. While RCSLS is the best-performing model in the BLI task, it shows suboptimal performance on XNLI across the board. This indicates

\footnotetext{
${ }^{11}$ Since our aim is to compare different bilingual spacesinput vectors for ESIM, kept fixed during training - we simply use the default ESIM hyper-parameter configuration.

${ }^{12}$ Note that one is able to use exactly the same ESIM model for all direct-projection CLE models (i.e., those not changing $\mathrm{L} 2$ vectors, $\mathbf{W}_{L 2}=I$ ) by using EN as L2, since different CLE models only produce different $\mathrm{L} 1$ vectors after projection. Exceptions are VECMAP and DLV models which transform the embeddings in L2 as well: for these models we had to train a different ESIM model for each language pair.

${ }^{13}$ The goal of this evaluation is not to compete with current state-of-the-art systems for (X)NLI (Artetxe and Schwenk, 2018; Lample and Conneau, 2019), but rather to provide means to analyze properties and relative performance of diverse CLE architectures in a downstream understanding task.
}

that specializing/overfitting CLE spaces to word translation may seriously hurt cross-lingual transfer for language understanding tasks. As the second indication of the mismatch, the unsupervised VECMAP model, outperformed by supervised models on BLI, performs on par with PROC and PROC$\mathrm{B}$ on XNLI. Finally, there are significant differences between BLI and XNLI performance across language pairs for most models-while we observe drastically better BLI performance for EN-DE and EN-FR compared to EN-RU and especially EN-TR, XNLI performance of most models for EN-RU and EN-TR is better than for EN-FR and close to that for EN-DE. While this can also be an artefact of the XNLI dataset creation, we support these individual observations by measuring an overall correlation (Spearman's $\rho$ ) of only 0.13 between corresponding BLI and XNLI scores over individual language-pair scores (for all models).

The PROC model performs significantly better on XNLI when trained on $5 \mathrm{~K}$ pairs than with $1 \mathrm{~K}$ pairs, and this is consistent with BLI results. However, we show that we can reach the same performance level using $1 \mathrm{~K}$ pairs and our proposed PROC-B bootstrapping scheme. VECMAP is again the most robust and most effective unsupervised model, but it is outperformed by the PROC-B model on more distant language pairs: EN-TR and EN-RU.

\subsection{Document Classification}

Evaluation Setup. We next evaluate CLEs on the cross-lingual document classification (CLDC) task. We use the TED CLDC corpus compiled by Hermann and Blunsom (2014). It includes 15 different topics and 12 language pairs (with EN as one of the languages in all pairs). A binary classifier is trained and evaluated for each topic and each language pair, using predetermined train and test splits. The intersection between TED languages and our BLI languages results in five CLDC evaluation pairs: EN-DE, EN-FR, EN-IT, EN-RU, and EN-TR. Since our goal is to compare different CLEs and isolate 


\begin{tabular}{lccccccc}
\hline Model & Dict & DE & FR & IT & RU & TR & Avg \\
\hline \multicolumn{7}{l}{ Supervised } \\
\hline PROC & $1 \mathrm{~K}$ & .250 & .107 & .158 & .127 & .309 & .190 \\
PROC & $5 \mathrm{~K}$ & .345 & .239 & .310 & .251 & .190 & .267 \\
PROC-B & $1 \mathrm{~K}$ & .374 & .182 & .205 & .243 & .254 & .251 \\
PROC-B & $3 \mathrm{~K}$ & .352 & .210 & .218 & .186 & .310 & .255 \\
DLV & $5 \mathrm{~K}$ & .299 & .175 & .234 & .375 & .208 & .258 \\
RCSLS & $1 \mathrm{~K}$ & .557 & .550 & .516 & .466 & .419 & $\mathbf{. 5 0 1}$ \\
RCSLS & $5 \mathrm{~K}$ & .588 & .540 & .451 & .527 & .447 & $\mathbf{. 5 1 0}$ \\
\hline Unupervised & & & & & & \\
\hline VECMAP & - & .433 & .316 & .333 & .504 & .439 &. $\mathbf{4 0 5}$ \\
MUSE & - & .288 & .223 & .198 & $.226^{*}$ & $.264^{*}$ & .240 \\
ICP & - & .492 & .254 & .457 & .362 & $.175^{*}$ & .348 \\
GWA & - & $.180^{*}$ & $.209^{*}$ & $.206^{*}$ & $.151^{*}$ & $.173^{*}$ & .184 \\
\hline
\end{tabular}

guage understanding than XNLI (which requires modeling of subtle nuances in sentence meaning).

Evaluation Setup. We employ a simple yet effective unsupervised CLIR model from Litschko et al. $(2018)^{15}$ that (1) builds query and document representations as weighted averages of word embeddings from a shared cross-lingual space and (2) computes the relevance score simply as the cosine similarity between aggregate query and document vectors. We evaluate the models on the standard test collections from the CLEF 2000-2003 ad-hoc retrieval Test Suite, ${ }^{16}$ again using the intersection with BLI languages. We preprocessed the test collections by lowercasing queries and documents and removing punctuation and single-character tokens.

Results and Discussion. CLIR MAP scores for nine language pairs are summarized in Table 5. The supervised Procrustes-based methods (PROC, PROC-B, and DLV) appear to have an edge in CLIR, with our bootstrapping PROC-B model outperforming all other CLE methods. ${ }^{17}$ Contrary to other downstream tasks, VECMAP is not the best-performing unsupervised model on CLIR - ICP significantly outperforms VECMAP (and also MUSE and GWA). The best-performing BLI model, RCSLS, displays poor CLIR performance, significantly worse than the simple PROC model.

\subsection{Further Discussion}

At first glance BLI performance shows only a weak and inconsistent correlation with results in downstream tasks. The behaviour of RCSLS is especially peculiar: it is the best-performing BLI model and it achieves the best results on CLDC by a wide margin, but it is not at all competitive on XNLI and falls short of other supervised models in the CLIR task. Trends in downstream results for other models (i.e., excluding RCSLS) seem to roughly correspond to trends in BLI scores.

To further investigate this, in Table 6 we measure correlations (in terms of Pearson's $\rho$ ) between aggregate task performances on BLI and three downstream tasks by considering (1) all models and

\footnotetext{
${ }^{15}$ The model is dubbed BWE-AGG in the original paper.

${ }^{16}$ http: //catalog.elra.info/en-us/ repository/browse/ELRA-E0008/

${ }^{17}$ Similarly to the BLI evaluation, we test the significance by applying a Student's t-test on two lists of ranks of relevant documents (concatenated across all test collections), produced by two models under comparison. Even with Bonferroni correction for multiple tests, PROC-B significantly outperforms all other CLE models at $\alpha=0.05$.
}

\footnotetext{
${ }^{14}$ We implemented a convolutional network with a single 1-D convolutional layer (with number of parameters fixed to fizers for each of the sizes $\{2,3,4,5\}$ ) and a max poot rithm (Kingma and $\mathrm{Ba}, 2014$ ).
} 


\begin{tabular}{llllllllllll}
\hline Model & Dict & DE-FI & DE-IT & DE-RU & EN-DE & EN-FI & EN-IT & EN-RU & FI-IT & FI-RU & Avg \\
\hline \multicolumn{1}{l}{ Supervised } & & & & & & & & & & & \\
\hline PROC & $1 \mathrm{~K}$ & 0.147 & 0.155 & 0.098 & 0.175 & 0.101 & 0.210 & 0.104 & 0.113 & 0.096 & 0.133 \\
PROC & $5 \mathrm{~K}$ & 0.255 & 0.212 & 0.152 & 0.261 & 0.200 & 0.240 & 0.152 & 0.149 & 0.146 & 0.196 \\
PROC-B & $1 \mathrm{~K}$ & 0.294 & 0.230 & 0.155 & 0.288 & 0.258 & 0.265 & 0.166 & 0.151 & 0.136 & $\mathbf{0 . 2 1 6}$ \\
PROC-B & 3K & 0.305 & 0.232 & 0.143 & 0.238 & 0.267 & 0.269 & 0.150 & 0.163 & 0.170 & $\mathbf{0 . 2 1 5}$ \\
DLV & 5K & 0.255 & 0.210 & 0.155 & 0.260 & 0.206 & 0.240 & 0.151 & 0.147 & 0.147 & 0.197 \\
RCSLS & $1 \mathrm{~K}$ & 0.114 & 0.133 & 0.077 & 0.163 & 0.063 & 0.163 & 0.106 & 0.074 & 0.069 & 0.107 \\
RCSLS & $5 \mathrm{~K}$ & 0.196 & 0.189 & 0.122 & 0.237 & 0.127 & 0.210 & 0.133 & 0.130 & 0.113 & 0.162 \\
\hline Unupervised & & & & & & & & & & & \\
\hline VECMAP & - & 0.240 & 0.129 & 0.162 & 0.200 & 0.150 & 0.201 & 0.104 & 0.096 & 0.109 & 0.155 \\
MuSE & - & $0.001 *$ & 0.210 & 0.195 & 0.280 & $0.000^{*}$ & 0.272 & $0.002^{*}$ & $0.002^{*}$ & $0.001^{*}$ & 0.107 \\
ICP & - & 0.252 & 0.170 & 0.167 & 0.230 & 0.230 & 0.231 & 0.119 & 0.117 & 0.124 & $\mathbf{0 . 1 8 2}$ \\
GWA & - & 0.218 & 0.139 & 0.149 & 0.013 & $0.005^{*}$ & $0.007 *$ & $0.005^{*}$ & 0.058 & 0.052 & 0.072 \\
\hline
\end{tabular}

Table 5: CLIR performance (MAP) of CLE models (the first language in each column is the query language, the second is the language of the document collection). Numbers in bold denote the best scores in the model group. Asterisks denote language pairs for which CLE models did not yield successful runs in BLI evaluation.

\begin{tabular}{lccc}
\hline Models & XNLI & CLDC & CLIR \\
\hline All models & 0.269 & 0.390 & 0.764 \\
All w/o RCSLS & $\mathbf{0 . 9 5 1}$ & 0.266 & $\mathbf{0 . 9 1 0}$ \\
\hline
\end{tabular}

Table 6: Correlations of model-level results between BLI and each of the three downstream tasks.

(2) all models except RCSLS. ${ }^{18}$ Without RCSLS, BLI results correlate almost perfectly with the results on XNLI and CLIR, while they correlate weakly with CLDC results.

The question is: why does RCSLS diverge from other models? All other models except RCSLS in the final step induce an orthogonal projection matrix using external or automatically induced translation dictionaries, minimizing the Euclidean distances between aligned words. In contrast, in order to maximize the CSLS similarity between aligned words, RCSLS relaxes the orthogonality condition imposed on the projection matrix $\mathbf{W}_{L 1}$. This allows for distortions of the source embedding space after projection. The exact nature of these distortions and their impact on downstream performance of RCSLS require further investigation. However, these findings indicate that downstream evaluation is even more important for CLE models that learn non-orthogonal projections. For CLE models with orthogonal projections, downstream results seems to be more in line with BLI performance.

The brief task correlation analysis is based on coarse-grained model-level aggregation. The actual selection of the strongest baseline models requires finer-grained tuning at the level of particu-

\footnotetext{
${ }^{18}$ For measuring correlation between BLI and each downstream task T, we average model's BLI performance only over the language pairs included in the task $\mathrm{T}$.
}

lar language pairs and evaluation tasks. However, our experiments also detect two robust baselines that should be included as indicative reference points in future research: PROC-B (supervised) and VECMAP (unsupervised).

\section{Conclusion}

The rapid progress in cross-lingual word embedding (CLE) methodology is currently not matched with the adequate progress in their fair and systematic evaluation and comparative analyses. CLE models are typically evaluated on a single task only: bilingual lexicon induction (BLI), and even the BLI task includes a wide variety of evaluation setups which are not directly comparable and thus hinder our ability to correctly interpret and generalize the key results. In this work, we have made the first step towards a comprehensive evaluation of cross-lingual word embeddings. By conducting a systematic evaluation of CLE models on a large number of language pairs in the BLI task and three downstream tasks, we have shed new light on the ability of current cutting-edge CLE models to support cross-lingual NLP. In particular, we have empirically proven that the quality of CLE models is largely task-dependent and that overfitting the models to the BLI task can result in deteriorated performance in downstream tasks. We have also highlighted the most robust supervised and unsupervised CLE models and have exposed the need for reassessing existing baselines, as well as for unified and comprehensive evaluation protocols. We hope that this study will encourage future work on CLE evaluation and analysis, and also assist in guiding the development of new CLE models. 


\section{References}

Jean Alaux, Edouard Grave, Marco Cuturi, and Armand Joulin. 2019. Unsupervised hyperalignment for multilingual word embeddings. In Proceedings of ICLR.

David Alvarez-Melis and Tommi Jaakkola. 2018. Gromov-Wasserstein alignment of word embedding spaces. In Proceedings of EMNLP, pages 18811890.

Waleed Ammar, George Mulcaire, Yulia Tsvetkov, Guillaume Lample, Chris Dyer, and Noah A Smith. 2016. Massively multilingual word embeddings. arXiv preprint arXiv:1602.01925.

Mikel Artetxe, Gorka Labaka, and Eneko Agirre. 2017. Learning bilingual word embeddings with (almost) no bilingual data. In Proceedings of $A C L$, pages $451-462$.

Mikel Artetxe, Gorka Labaka, and Eneko Agirre. 2018a. Generalizing and improving bilingual word embedding mappings with a multi-step framework of linear transformations. In Proceedings of AAAI, pages 5012-5019.

Mikel Artetxe, Gorka Labaka, and Eneko Agirre. 2018b. A robust self-learning method for fully unsupervised cross-lingual mappings of word embeddings. In Proceedings of ACL, pages 789-798.

Mikel Artetxe, Gorka Labaka, Eneko Agirre, and Kyunghyun Cho. 2018c. Unsupervised neural machine translation. In Proceedings of ICLR.

Mikel Artetxe and Holger Schwenk. 2018. Massively multilingual sentence embeddings for zeroshot cross-lingual transfer and beyond. CoRR, abs/1812.10464.

Anthony Bell and Terrence Sejnowski. 1997. The 'Independent Components' of Natural Scenes are Edge Filters. Vision Research.

Piotr Bojanowski, Edouard Grave, Armand Joulin, and Tomas Mikolov. 2017. Enriching word vectors with subword information. Transactions of the ACL, 5:135-146.

Samuel R. Bowman, Gabor Angeli, Christopher Potts, and Christopher D Manning. 2015. A large annotated corpus for learning natural language inference. In Proceedings of EMNLP, pages 632-642.

Qian Chen, Xiaodan Zhu, Zhen-Hua Ling, Si Wei, Hui Jiang, and Diana Inkpen. 2017. Enhanced LSTM for natural language inference. In Proceedings of $A C L$, pages $1657-1668$.

Xilun Chen and Claire Cardie. 2018. Unsupervised multilingual word embeddings. In Proceedings of EMNLP, pages 261-270.
Alexis Conneau, Guillaume Lample, Marc'Aurelio Ranzato, Ludovic Denoyer, and Hervé Jégou. 2018a. Word translation without parallel data. In Proceedings of ICLR.

Alexis Conneau, Ruty Rinott, Guillaume Lample, Adina Williams, Samuel Bowman, Holger Schwenk, and Veselin Stoyanov. 2018b. XNLI: Evaluating cross-lingual sentence representations. In Proceedings of EMNLP, pages 2475-2485.

Zi-Yi Dou, Zhi-Hao Zhou, and Shujian Huang. 2018 Unsupervised bilingual lexicon induction via latent variable models. In Proceedings of EMNLP, pages 621-626.

Yerai Doval, Jose Camacho-Collados, Luis Espinosa Anke, and Steven Schockaert. 2018. Improving cross-lingual word embeddings by meeting in the middle. In Proceedings of EMNLP, pages 294-304.

Rotem Dror, Gili Baumer, Segev Shlomov, and Roi Reichart. 2018. The hitchhiker's guide to testing statistical significance in natural language processing. In Proceedings of ACL, pages 1383-1392.

Manaal Faruqui and Chris Dyer. 2014. Improving vector space word representations using multilingual correlation. In Proceedings of the 14th Conference of the European Chapter of the Association for Computational Linguistics, pages 462-471.

Ian J. Goodfellow, Jean Pouget-Abadie, Mehdi Mirza, Bing Xu, David Warde-Farley, Sherjil Ozair, Aaron C. Courville, and Yoshua Bengio. 2014. Generative adversarial nets. In Proceedings of NIPS, pages 2672-2680.

Stephan Gouws, Yoshua Bengio, and Greg Corrado. 2015. BilBOWA: Fast bilingual distributed representations without word alignments. In Proceedings of ICML, pages 748-756.

Edouard Grave, Armand Joulin, and Quentin Berthet. 2018. Unsupervised alignment of embeddings with Wasserstein procrustes. CoRR, abs/1805.11222.

Jiang Guo, Wanxiang Che, David Yarowsky, Haifeng Wang, and Ting Liu. 2015. Cross-lingual dependency parsing based on distributed representations. In Proceedings of ACL, pages 1234-1244.

Bradley Hauer, Garrett Nicolai, and Grzegorz Kondrak. 2017. Bootstrapping unsupervised bilingual lexicon induction. In Proceedings of EACL, pages 619-624.

Karl Moritz Hermann and Phil Blunsom. 2014. Multilingual models for compositional distributed semantics. In Proceedings of ACL, pages 58-68.

Geert Heyman, Ivan Vulić, and Marie-Francine Moens. 2017. Bilingual lexicon induction by learning to combine word-level and character-level representations. In Proceedings of EACL, pages 1085-1095. 
Yedid Hoshen and Lior Wolf. 2018. Non-adversarial unsupervised word translation. In Proceedings of EMNLP, pages 469-478.

Kejun Huang, Matt Gardner, Evangelos Papalexakis, Christos Faloutsos, Nikos Sidiropoulos, Tom Mitchell, Partha P. Talukdar, and Xiao Fu. 2015. Translation invariant word embeddings. In Proceedings of EMNLP, pages 1084-1088.

Roy Jonker and Anton Volgenant. 1987. A shortest augmenting path algorithm for dense and sparse linear assignment problems. Computing, 38(4):325340.

Armand Joulin, Piotr Bojanowski, Tomas Mikolov, Hervé Jégou, and Edouard Grave. 2018. Loss in translation: Learning bilingual word mapping with a retrieval criterion. In Proceedings of EMNLP, pages 2979-2984.

Yunsu Kim, Jiahui Geng, and Hermann Ney. 2018 Improving unsupervised word-by-word translation with language model and denoising autoencoder. In Proceedings of EMNLP, pages 862-868.

Diederik P Kingma and Jimmy Ba. 2014. Adam: A method for stochastic optimization. arXiv preprint arXiv:1412.6980.

Alexandre Klementiev, Ivan Titov, and Binod Bhattarai 2012. Inducing crosslingual distributed representations of words. Proceedings of COLING, pages 1459-1474.

Guillaume Lample and Alexis Conneau. 2019. Crosslingual language model pretraining. CoRR, abs/1901.07291.

Guillaume Lample, Myle Ott, Alexis Conneau, Ludovic Denoyer, and Marc'Aurelio Ranzato. 2018. Phrase-based \& neural unsupervised machine translation. In Proceedings of EMNLP, pages 50395049 .

Omer Levy, Anders Søgaard, and Yoav Goldberg. 2017. A strong baseline for learning cross-lingual word embeddings from sentence alignments. In Proceedings of EACL, pages 765-774.

Robert Litschko, Goran Glavaš, Simone Paolo Ponzetto, and Ivan Vulić. 2018. Unsupervised crosslingual information retrieval using monolingual data only. In Proceedings of SIGIR, pages 1253-1256.

Thang Luong, Hieu Pham, and Christopher D. Manning. 2015. Bilingual word representations with monolingual quality in mind. In Proceedings of the 1 st Workshop on Vector Space Modeling for Natural Language Processing, pages 151-159.

Stephen Mayhew, Chen-Tse Tsai, and Dan Roth. 2017. Cheap translation for cross-lingual named entity recognition. In Proceedings of EMNLP, pages 2536-2545.
Tomas Mikolov, Quoc V Le, and Ilya Sutskever. 2013. Exploiting similarities among languages for machine translation. CoRR, abs/1309.4168.

Tanmoy Mukherjee, Makoto Yamada, and Timothy Hospedales. 2018. Learning unsupervised word translations without adversaries. In Proceedings of EMNLP, pages 627-632.

Ndapa Nakashole. 2018. NORMA: Neighborhood sensitive maps for multilingual word embeddings. In Proceedings of EMNLP, pages 512-522.

Gabriel Peyré, Marco Cuturi, and Justin Solomon. 2016. Gromov-Wasserstein averaging of kernel and distance matrices. In Proceedings of ICML, pages 2664-2672.

Sebastian Ruder, Ryan Cotterell, Yova Kementchedjhieva, and Anders Søgaard. 2018a. A discriminative latent-variable model for bilingual lexicon induction. In Proceedings of EMNLP, pages 458-468.

Sebastian Ruder, Anders Søgaard, and Ivan Vulić. 2018b. A survey of cross-lingual embedding models. arXiv preprint arXiv:1706.04902.

Peter H Schönemann. 1966. A generalized solution of the orthogonal Procrustes problem. Psychometrika, 31(1):1-10.

Samuel L. Smith, David H.P. Turban, Steven Hamblin, and Nils Y. Hammerla. 2017. Offline bilingual word vectors, orthogonal transformations and the inverted softmax. In Proceedings of ICLR.

Anders Søgaard, Željko Agić, Héctor Martínez Alonso, Barbara Plank, Bernd Bohnet, and Anders Johannsen. 2015. Inverted indexing for cross-lingual NLP. In Proceedings of ACL, pages 1713-1722.

Anders Søgaard, Sebastian Ruder, and Ivan Vulić. 2018. On the limitations of unsupervised bilingual dictionary induction. In Proceedings of ACL, pages 778788.

Shyam Upadhyay, Manaal Faruqui, Chris Dyer, and Dan Roth. 2016. Cross-lingual models of word embeddings: An empirical comparison. In Proceedings of $A C L$, pages 1661-1670.

Ivan Vulić and Anna Korhonen. 2016. On the role of seed lexicons in learning bilingual word embeddings. In Proceedings of ACL, pages 247-257.

Ivan Vulić and Marie-Francine Moens. 2015. Monolingual and Cross-Lingual Information Retrieval Models Based on (Bilingual) Word Embeddings. In Proceedings of the 38th International ACM SIGIR Conference on Research and Development in Information Retrieval (SIGIR), pages 363-372.

Ivan Vulić and Marie-Francine Moens. 2016. Bilingual distributed word representations from documentaligned comparable data. Journal of Artificial Intelligence Research, 55:953-994. 
Søren Wichmann, André Müller, Viveka Velupillai, Cecil H Brown, Eric W Holman, Pamela Brown, Sebastian Sauppe, Oleg Belyaev, Matthias Urban, Zarina Molochieva, et al. 2018. The asjp database (version 18).

Adina Williams, Nikita Nangia, and Samuel R. Bowman. 2018. A broad-coverage challenge corpus for sentence understanding through inference. In Proceedings of the 2018 Conference of the North American Chapter of the Association for Computational Linguistics: Human Language Technologies, Volume 1 (Long Papers), volume 1, pages 1112-1122.

Chao Xing, Dong Wang, Chao Liu, and Yiye Lin. 2015. Normalized word embedding and orthogonal transform for bilingual word translation. In Proceedings of the 2015 Conference of the North American Chapter of the Association for Computational Linguistics. Human Language Technologies, pages 1006-1011.

Ruochen $\mathrm{Xu}$, Yiming Yang, Naoki Otani, and Yuexin Wu. 2018. Unsupervised cross-lingual transfer of word embedding spaces. In Proceedings of the 2018 Conference on Empirical Methods in Natural Language Processing, pages 2465-2474. Association for Computational Linguistics.

Alexander Yeh. 2000. More accurate tests for the statistical significance of result differences. In Proceedings of the 18th conference on Computational linguistics-Volume 2, pages 947-953. Association for Computational Linguistics.

Meng Zhang, Yang Liu, Huanbo Luan, and Maosong Sun. 2017. Adversarial training for unsupervised bilingual lexicon induction. In Proceedings of $A C L$, volume 1, pages 1959-1970.

Yuan Zhang, David Gaddy, Regina Barzilay, and Tommi Jaakkola. 2016. Ten pairs to tag - Multilingual POS tagging via coarse mapping between embeddings. In Proceedings of the 2016 Conference of the North American Chapter of the Association for Computational Linguistics: Human Language Technologies, pages 1307-1317.

Will Y Zou, Richard Socher, Daniel Cer, and Christopher D Manning. 2013. Bilingual word embeddings for phrase-based machine translation. In Proceedings of the 2013 Conference on Empirical Methods in Natural Language Processing, pages 1393-1398. 


\begin{tabular}{|c|c|c|c|c|c|c|c|c|c|c|c|c|c|c|c|}
\hline Model & Dict & DE-FI & DE-FR & DE-HR & DE-IT & DE-RU & DE-TR & EN-DE & EN-FI & EN-FR & EN-HR & EN-IT & EN-RU & EN-TR & FI-FR \\
\hline $\mathrm{CCA}$ & $1 \mathrm{~K}$ & 241 & 0.422 & 0.206 & 0.414 & 0.308 & 0.153 & 0.458 & 0.259 & 0.582 & 0.218 & 0.538 & 0.336 & 0.218 & 0.230 \\
\hline $\mathrm{CCA}$ & $3 \mathrm{~K}$ & 0.328 & 0.494 & 0.298 & 0.491 & 0.399 & 0.251 & 0.531 & 0.351 & 0.642 & 0.299 & 0.613 & 0.434 & 0.314 & 0.332 \\
\hline $\mathrm{CCA}$ & $5 \mathrm{~K}$ & 0.353 & 0.509 & 0.318 & 0.506 & 0.411 & 0.280 & 0.542 & 0.383 & 0.652 & 0.325 & 0.624 & 0.454 & 0.327 & 0.362 \\
\hline PROC & $1 \mathrm{~K}$ & 0.264 & 0.428 & 0.225 & 0.421 & 0.323 & 0.169 & 0.458 & 0.271 & 0.579 & 0.225 & 0.535 & 0.352 & 0.225 & 0.239 \\
\hline PROC & $3 \mathrm{~K}$ & 0.340 & 0.499 & 0.308 & 0.495 & 0.413 & 0.260 & 0.532 & 0.365 & 0.642 & 0.307 & 0.611 & 0.449 & 0.320 & 0.333 \\
\hline PROC & $5 \mathrm{~K}$ & 0.359 & 0.511 & 0.329 & 0.510 & 0.425 & 0.284 & 0.544 & 0.396 & 0.654 & 0.336 & 0.625 & 0.464 & 0.335 & 0.362 \\
\hline PROC-B & $1 \mathrm{~K}$ & 0.354 & 0.511 & 0.306 & 0.507 & 0.392 & 0.250 & 0.521 & 0.360 & 0.633 & 0.296 & 0.605 & 0.419 & 0.301 & 0.329 \\
\hline PROC-B & $3 \mathrm{~K}$ & 0.362 & 0.514 & 0.324 & 0.508 & 0.413 & 0.278 & 0.532 & 0.380 & 0.642 & 0.336 & 0.612 & 0.449 & 0.328 & 0.350 \\
\hline DLV & $1 \mathrm{~K}$ & 0.259 & 0.384 & 0.222 & 0.420 & 0.325 & 0.167 & 0.454 & 0.271 & 0.546 & 0.225 & 0.537 & 0.353 & 0.221 & 0.209 \\
\hline DLV & $3 \mathrm{~K}$ & 0.341 & 0.496 & 0.306 & 0.494 & 0.411 & 0.261 & 0.533 & 0.365 & 0.636 & 0.307 & 0.611 & 0.444 & 0.320 & 0.321 \\
\hline DLV & $5 \mathrm{~K}$ & 0.357 & 0.506 & 0.328 & 0.510 & 0.423 & 0.284 & 0.545 & 0.396 & 0.649 & 0.334 & 0.625 & 0.467 & 0.335 & 0.351 \\
\hline RCSLS & $1 \mathrm{~K}$ & 0.288 & 0.459 & 0.262 & 0.453 & 0.361 & 0.201 & 0.501 & 0.306 & 0.612 & 0.267 & 0.565 & 0.401 & 0.275 & 0.269 \\
\hline RCSLS & $3 \mathrm{~K}$ & 0.373 & 0.524 & 0.337 & 0.518 & 0.442 & 0.296 & 0.568 & 0.404 & 0.665 & 0.357 & 0.637 & 0.491 & 0.364 & 0.367 \\
\hline RCSLS & $5 \mathrm{~K}$ & 0.395 & 0.536 & 0.359 & 0.529 & 0.458 & 0.324 & 0.580 & 0.438 & 0.675 & 0.375 & 0.652 & 0.510 & 0.386 & 0.395 \\
\hline VECMAP & - & 0.302 & 0.505 & 0.300 & 0.493 & 0.322 & 0.253 & 0.521 & 0.292 & 0.626 & 0.268 & 0.600 & 0.323 & 0.288 & 0.368 \\
\hline MUSE & - & 0.000 & 0.005 & 0.245 & 0.496 & 0.272 & 0.237 & 0.520 & 0.000 & 0.632 & 0.000 & 0.608 & 0.000 & 0.294 & 0.348 \\
\hline ICP & - & 0.251 & 0.454 & 0.240 & 0.447 & 0.245 & 0.215 & 0.486 & 0.262 & 0.613 & 0.000 & 0.577 & 0.259 & 0.000 & 0.000 \\
\hline GWA & - & 0.216 & 0.433 & 0.015 & 0.440 & 0.222 & 0.101 & 0.029 & 0.022 & 0.462 & 0.003 & 0.017 & 0.002 & 0.003 & 0.121 \\
\hline
\end{tabular}

Table 7: Appendix: BLI performance (MAP) for first batch (14) of language pairs.

\begin{tabular}{|c|c|c|c|c|c|c|c|c|c|c|c|c|c|c|c|}
\hline Model & Dict & FI-HR & FI-IT & FI-RU & HR-FR & HR-IT & HR-RU & IT-FR & RU-FR & RU-IT & TR-FI & TR-FR & TR-HR & TR-IT & TR-RU \\
\hline CCA & $1 \mathrm{~K}$ & 0.167 & 0.232 & 0.214 & 0.238 & 0.240 & 0.256 & 0.612 & 0.344 & 0.352 & 0.151 & 0.213 & 0.134 & 0.202 & 0.146 \\
\hline CCA & $3 \mathrm{~K}$ & 0.264 & 0.328 & 0.306 & 0.346 & 0.345 & 0.348 & 0.659 & 0.452 & 0.449 & 0.232 & 0.308 & 0.211 & 0.309 & \\
\hline CCA & $5 \mathrm{~K}$ & 0.288 & 0.353 & 0.340 & 0.372 & 0.366 & 0.367 & 0.668 & 0.469 & 0.474 & 0.260 & 0.337 & 0.250 & 0.331 & 0.285 \\
\hline PROC & $1 \mathrm{~K}$ & 0.187 & 0.247 & 0.233 & 0.248 & 0.247 & 0.269 & 0.615 & 0.352 & 0.360 & 0.169 & 0.215 & 0.148 & 0.211 & 0.168 \\
\hline PROC & $3 \mathrm{~K}$ & 0.269 & 0.328 & 0.310 & 0.346 & 0.350 & 0.353 & 0.659 & 0.455 & 0.455 & 0.241 & 0.312 & 0.219 & 0.312 & 0.262 \\
\hline PROC & $5 \mathrm{~K}$ & 0.294 & 0.355 & 0.342 & 0.374 & 0.364 & 0.372 & 0.669 & 0.470 & 0.474 & 0.269 & 0.338 & 0.259 & 0.335 & 0.290 \\
\hline PROC-B & $1 \mathrm{~K}$ & 0.263 & 0.328 & 0.315 & 0.335 & 0.343 & 0.348 & 0.665 & 0.467 & 0.466 & 0.247 & 0.305 & 0.210 & 0.298 & 0.230 \\
\hline PROC-B & $3 \mathrm{~K}$ & 0.293 & 0.348 & 0.327 & 0.365 & 0.368 & 0.365 & 0.664 & 0.478 & 0.476 & 0.270 & 0.333 & 0.244 & 0.330 & 0.262 \\
\hline DLV & $1 \mathrm{~K}$ & 0.184 & 0.244 & 0.225 & 0.214 & 0.245 & 0.264 & 0.585 & 0.320 & 0.358 & 0.161 & 0.194 & 0.144 & 0.209 & 0.161 \\
\hline DLV & $3 \mathrm{~K}$ & 0.269 & 0.331 & 0.307 & 0.331 & 0.348 & 0.353 & 0.653 & 0.446 & 0.452 & 0.243 & 0.306 & 0.219 & 0.311 & 0.261 \\
\hline DLV & $5 \mathrm{~K}$ & 0.294 & 0.356 & 0.342 & 0.364 & 0.366 & 0.374 & 0.665 & 0.466 & 0.475 & 0.268 & 0.333 & 0.255 & 0.336 & 0.289 \\
\hline RCSLS & $1 \mathrm{~K}$ & 0.214 & 0.272 & 0.257 & 0.281 & 0.275 & 0.291 & 0.637 & 0.381 & 0.383 & 0.194 & 0.247 & 0.170 & 0.246 & 0.191 \\
\hline RCSLS & $3 \mathrm{~K}$ & 0.296 & 0.362 & 0.341 & 0.384 & 0.382 & 0.379 & 0.673 & 0.477 & 0.472 & & 0.348 & 0.256 & 0.340 & 0.290 \\
\hline RCSLS & $5 \mathrm{~K}$ & 0.321 & 0.388 & 0.376 & 0.412 & 0.399 & 0.404 & 0.682 & 0.494 & 0.491 & 0.300 & 0.375 & 0.285 & 0.368 & 0.324 \\
\hline VECMAP & - & 0.280 & 0.355 & 0.312 & 0.402 & 0.389 & 0.376 & 0.667 & 0.463 & 0.463 & 0.246 & 0.341 & 0.223 & 0.332 & 0.200 \\
\hline MUSE & - & 0.228 & 0.000 & 0.001 & 0.000 & 0.000 & 0.000 & 0.662 & 0.005 & 0.450 & 0.000 & 0.000 & 0.133 & 0.000 & 0.000 \\
\hline ICP & - & 0.208 & 0.263 & 0.231 & 0.282 & 0.045 & 0.309 & 0.629 & 0.000 & 0.394 & 0.173 & 0.000 & 0.138 & & 0.119 \\
\hline GWA & - & 0.009 & 0.173 & 0.086 & 0.018 & 0.021 & 0.001 & 0.655 & 0.188 & 0.190 & 0.102 & 0.106 & 0.016 & 0.142 & 0.040 \\
\hline
\end{tabular}

Table 8: Appendix: BLI performance (MAP) for the second batch (14) of language pairs. 